\title{
Stimulated terahertz emission due to electronic Raman scattering in silicon
}

\author{
S. G. Pavlov ${ }^{1}$, H.-W. Hübers ${ }^{1}$, U. Böttger ${ }^{1}$, J. N. Hovenier ${ }^{2}$, N. V. Abrosimov ${ }^{3}$, H. Riemann ${ }^{3}$, R. \\ Kh. Zhukavin ${ }^{4}$, V. N. Shastin ${ }^{4}$, B. Redlich ${ }^{5}$ and A. F. G. van der Meer ${ }^{5}$ \\ ${ }^{1}$ Institute of Planetary Research, German Aerospace Center (DLR), 12489 Berlin, Germany \\ ${ }^{2}$ Kavli Institute of Nanoscience Delft, Delft University of Technology, 2600 GA Delft, The Netherlands \\ ${ }^{3}$ Leibniz Institute of Crystal Growth, Max-Born-Straße 2, 12489 Berlin, Germany \\ ${ }^{4}$ Institute for Physics of Microstructures, Russian Academy of Sciences, Nizhny Novgorod, Russia \\ ${ }^{5}$ FOM-Institute for Plasma Physics, 3439 MN Nieuwegein, The Netherlands
}

$$
\text { sergeij.pavlov@dlr.de }
$$

Silicon-based semiconductors are intensively investigated over the past years as promising candidates for optoelectronic devices at terahertz (THz) frequencies [1]. Optically pumped intracenter silicon lasers, realized in the past decade in the $\mathrm{THz}$ range, are based on direct optical transitions between shallow levels of different shallow donors [2]. Recently, terahertz Raman laser emission has been demonstrated in silicon doped by antimony [3] and phosphorus [4].

We report on realization of terahertz lasers based on intracenter electronic Raman scattering in silicon doped by arsenic (Si:As, frequency range $4.8-5.1 \mathrm{THz}$ and $5.9-6.5 \mathrm{THz}$ ) and silicon doped by bismuth $(\mathrm{Si}: \mathrm{Bi}, 4.6-5.9 \mathrm{THz})$ under optical excitation by infrared frequency-tunable free electron laser at low lattice temperatures. The Stokes shift of the observed laser emission is equal to the Raman-active donor electronic transition between the ground $1 \mathrm{~s}\left(\mathrm{~A}_{1}\right)$ and the excited $1 \mathrm{~s}(\mathrm{E})$ donor states. Raman terahertz gain of the lasers is similar to those observed for the donor-type terahertz silicon donor lasers.

\section{References}

[1] A. Borak, "Towards bridging the terahertz gap with silicon-based lasers", Science, vol. 308, p. 6389 (2005).

[2] H.-W. Hübers, S. G. Pavlov and V. N. Shastin, "Terahertz lasers based on germanium and silicon", Semicond. Sci. Technol., vol. 20, p. S211 (2005).

[3] S. G. Pavlov, H.-W. Hübers, J .N. Hovenier, T. O. Klaassen, D. A. Carder, P. J. Phillips, B. Redlich, H. Riemann, R. Kh. Zhukavin, and V. N. Shastin, "Stimulated terahertz Stokes emission of silicon crystals doped with antimony donors", Phys. Rev. Lett., vol. 96, p. 037404 (2006).

[4] S. G. Pavlov, H.-W. Hübers, U. Böttger, R. Kh. Zhukavin, V. N. Shastin, J. N. Hovenier, B. Redlich, N. V. Abrosimov, and H. Riemann, “Terahertz Raman laser based on silicon doped with phosphorus”, Appl. Phys. Lett., vol. 92, p. 091111 (2008). 